\title{
Photon and dilepton production in soft wall AdS/QCD
}

A. Nata Atmaja and K. Schalm

\author{
Institute Lorentz for Theoretical Physics, ${ }^{1}$ \\ Niels Bohrweg 2, 2333CA Leiden, The Netherlands \\ Instituut voor Theoretische Fysica, \\ Valckenierstraat 65, 1018XE Amsterdam, The Netherlands \\ E-mail: ardian@lorentz.leidenuniv.nl, kschalm@lorentz.leidenuniv.nl
}

\begin{abstract}
We consider the Soft-Wall-model of AdS/QCD to calculate photon production in strongly coupled Quark Gluon Plasma (sQGP). The IR cut-off only affects the lowfrequency-component of the production rate. The full spectral function is determined numerically and shows remarkable similarity to computations of the photon production rate in AdS-duals of $\mathcal{N}=2$ theories with massive flavor. It is further support that SoftWall AdS-QCD correctly captures the IR physics of the chiral perturbation theory regime of QCD. We confirm this by relating the IR-effects of the massive flavor deformations to the AdS/QCD soft wall cut-off. The AdS/QCD spectral function is smooth, however, and unlike massive flavor models shows no spectral peaks.
\end{abstract}

KEYWords: Gauge-gravity correspondence, AdS-CFT Correspondence, Phenomenological Models, Hadronic Colliders

ARXiv EPRINT: 0802.1460

\footnotetext{
${ }^{1}$ Permanent address from September 1st 2007.
} 


\section{Contents}

$\begin{array}{llr}1 & \text { Introduction } & 1\end{array}$

2 Photon and dilepton production $\quad 2$

2.1 Photon and dilepton rates at strong coupling 3

$\begin{array}{lll}3 & \text { Solving the system } & 6\end{array}$

3.1 Lightlike momenta 6

3.1.1 Analytic solutions for lightlike momenta at low and high frequency 6

3.1.2 Numerical solution for lightlike momenta 9

$\begin{array}{ll}\text { 3.1.3 The spectral density for lightlike momenta } & 10\end{array}$

$\begin{array}{ll}3.2 & \text { Timelike and spacelike momenta } \\ & 11\end{array}$

$\begin{array}{lll}3.2 .1 & \text { Numerical solution for transverse scalar function } & 12\end{array}$

$\begin{array}{lll}3.2 .2 & \text { Numerical solution for longitudinal scalar function } & 12\end{array}$

3.2.3 The spectral density for time- and space-like momenta 13

$\begin{array}{lll}3.3 & \text { Electrical conductivity } & 13\end{array}$

4 Conclusion: soft wall cut-offs as an IR mass-gap 14

$\begin{array}{ll}\text { A Spectral function low frequency limit for lightlike momenta } & 17\end{array}$

$\begin{array}{ll}\text { B The susceptibility and the diffusion constant } & 18\end{array}$

\section{Introduction}

One of the current challenges in theoretical particle physics is to compute properties of the strongly coupled sQGP discovered at RHIC. AdS/CFT tools have given us some insight into the strongly coupled thermodynamics of gauge theories [1-7]. However, it remains a mystery why these, mostly $\mathcal{N}=4$ supersymmetric, YM calculations work well for QCD. Part of the challenge is to either understand why this is so, or to find AdS duals of theories resembling QCD closer than $\mathcal{N}=4$ SYM. In this latter context a phenomenological AdS dual to Chiral perturbation theory constructed by Erlich et.al. is perhaps a good candidate [8]. It predicts the meson spectrum within 10\%-15\% accuracy; and with a smooth instead of a hard IR-cutoff predicts the critical $T_{c}$ surprisingly well $[9,10] .{ }^{1}$

\footnotetext{
${ }^{1}$ The prediction of the critical deconfinement temperature in soft-wall model is really quite surprising; The neglection of backreaction invalidates the thermodynamic relations that are used to derived it. Detailed studies that include the effects of backreaction are e.g. [23-25] for the backgrounds which are believed to correspond to large $N_{c}$ pure Yang-Mills theory and $[26,27]$ for the backgrounds that mimic QCD equation of state.
} 
This IR-cutoff is the essential new ingredient in AdS/QCD compared to AdS/CFT. Here we shall investigate the effects of this cutoff on photon and dilepton production rates at strongly coupling. Remarkably the $\mathcal{N}=4 \mathrm{SYM}$ CFT computation of these production rates suggested they are not affected by a hard IR-cut-off even for temperatures infinitesimally above the cut-off [11]. Intuitively this seems rather strange. At energies and temperatures close the QCD scale IR effects should start to affect the production rate. We shall find that for smoothly IR-cutoff AdS/QCD this is indeed the case. The robustness of our phenomenological result of how photon production rates are effected by changing the IRcutoff is confirmed by a calculation by Mateos and Patiño $[12,13]$ of the photon production rate in AdS dual of a $\mathcal{N}=2$ theory with massive flavor. Here the flavor sector acts as the effective IR-cutoff, and we will be able to show this by relating the mass-parameter to the Soft-Wall cut-off scale. Soft-wall AdS/QCD is more crude than massive flavor models, of course, and this is evident in the lack of spectral peaks that we shall find.

Photon production in a medium such as sQGP was discussed in detail both from strong and weak coupling point view in [11]. We briefly review this in section 2 and show there how the strong coupling calculation is modified by considering AdS/QCD instead of pure $\mathcal{N}=4 \mathrm{SYM}$. In section 3 , we present our solution and discuss its results in section 4 with a comparison to photon production in AdS duals of $\mathcal{N}=2$ massive flavor theories.

\section{Photon and dilepton production}

One of the observational phenomena in RHIC is the spontaneous production of photons from the sQGP of hot charged particles. This direct photon spectrum ought to be a good probe of the strongly coupled quark-gluon soup, as the weakly interacting photons should escape nearly unaffected from the small finite size collision area [14].

As is described in [11], we can therefore regard the dynamically formed sQGP to first approximation as a field theory at finite temperature. For a standard perturbative electromagnetic current coupling $e J_{\mu}^{\mathrm{EM}} A^{\mu}$, the first order photon production rate is then given by $[11,15]$

$$
d \Gamma_{\gamma}=\left.\frac{d^{3} k}{(2 \pi)^{3} 2 k^{0}} e^{2} n_{B}\left(k^{0}\right) \eta^{\mu \nu} \chi_{\mu \nu}(K)\right|_{k^{0}=|\vec{k}|} .
$$

Here $K \equiv\left(k^{0}, \vec{k}\right)$ is a momentum 4-vector, $n_{B}\left(k^{0}\right)=1 /\left(e^{\beta k^{0}}-1\right)$ the Bose-Einstein distribution function, and the spectral density $\chi_{\mu \nu}(K)$ is proportional to the imaginary part of the (finite temperature) retarded current-current correlation function

$$
\begin{aligned}
\chi_{\mu \nu}(K) & =-2 \operatorname{Im}\left(G_{\mu \nu}^{R, \beta}(K)\right), \\
G_{\mu \nu}^{R, \beta}(K) & =\int d^{4} X e^{-i K \cdot X}\left\langle J_{\mu}^{\mathrm{EM}}(0) J_{\nu}^{\mathrm{EM}}(X)\right\rangle_{\beta} \theta\left(-x^{0}\right) .
\end{aligned}
$$

At finite temperature, Lorentz invariance is broken by the heat bath. We can use the remaining rotational symmetry plus gauge invariance to simplify the retarded correlator to

$$
G_{\mu \nu}^{R, \beta \neq 0}(K)=P_{\mu \nu}^{T}(K) \Pi^{T}(K)+P_{\mu \nu}^{L}(K) \Pi^{L}(K),
$$


Here the transverse and longitudinal projectors are $P_{00}^{T}(K)=0, P_{0 i}^{T}(K)=0, P_{i j}^{T}(K)=$ $\delta_{i j}-k_{i} k_{j} /|\vec{k}|^{2}$, and $P_{\mu \nu}^{L}(K)=P_{\mu \nu}(K)-P_{\mu \nu}^{T}(K)$, with $i, j=x, y, z$. We can trivially consider charged lepton production as well by considering non-lightlike momenta for offshell photons: The leptons then result from virtual photon decay. Lepton pair production for each lepton species in the leading order of the electromagnetic couplings $e$ and $e_{l}$, is given by $[11,15]$

$$
d \Gamma_{l \bar{l}}=\frac{d^{4} K}{(2 \pi)^{4}} \frac{e^{2} e_{l}^{2}}{6 \pi|K|^{5}}\left[-K^{2}-4 m^{2}\right]^{1 / 2}\left(-K^{2}+2 m^{2}\right) n_{b}\left(k^{0}\right) \chi_{\mu}^{\mu}(K) \theta\left(k^{0}\right) \theta\left(-K^{2}-4 m^{2}\right),
$$

with $e_{l}$ the electric charge of the lepton, $m$ the lepton mass, $\theta(x)$ a unit step function, and the spectral density $\chi_{\mu \nu}(K)$ is evaluated at the timelike momentum of the emitted particle pair. Note that both $\Pi^{T}$ and $\Pi^{L}$ contribute to the dilepton rate, but only $\Pi^{T}$ contributes to the photon emission rate, because the longitudinal part must vanish for lightlike momenta, i.e. the unphysical longitudinal mode is not a propagating degree of freedom.

Finally, fluctuation-dissipation relates the zero-frequency limit of the spectral density to the electrical conductivity $\sigma$ :

$$
\sigma=\lim _{k^{0} \rightarrow 0} \frac{e^{2}}{6 T} n_{B}\left(k_{0}\right) \eta^{\mu \nu} \chi_{\mu \nu}\left(k^{0}, \vec{k}=0\right),
$$

or, if $k_{\mu}$ is lightlike

$$
\sigma=\left.\lim _{k^{0} \rightarrow 0} \frac{e^{2}}{4 T} n_{B}\left(k_{0}\right) \eta^{\mu \nu} \chi_{\mu \nu}(K)\right|_{|\vec{k}|=k^{0}} .
$$

\subsection{Photon and dilepton rates at strong coupling}

The AdS/CFT dictionary gives that the large $N_{c}$ limit of strongly coupled $d=4 \mathcal{N}=4$ SYM theory at finite temperature $T$ has a dual description in terms of five dimensional AdS-supergravity in the background of a black hole [6]

$$
d s^{2}=\frac{(\pi T R)^{2}}{u}\left[-f(u) d t^{2}+d x^{2}+d y^{2}+d z^{2}\right]+\frac{R^{2}}{4 u^{2} f(u)} d u^{2} .
$$

Here $f(u)=1-u^{2}$, with $u \in[0,1]$ a dimensionless radial AdS coordinate related through $u=(\pi T z)^{2}$ to standard AdS coordinates, and $R$ is the curvature radius of the AdS space. ${ }^{2}$ The metric (2.7) has a horizon at $u=1$ with Hawking temperature $T$ and a boundary at $u=0$.

Qualitatively the same is expected hold for other 4-dim field theories. As a model for low energy QCD we shall take the AdS dual of chiral perturbation theory. This AdS/QCD consists of the fields $A_{L \mu}^{a}, A_{R \mu}^{a}$, dual to the $\mathrm{SU}\left(N_{f}\right)_{L} \times \mathrm{SU}\left(N_{f}\right)_{R}$ currents and a scalar $X$ dual to the quark condensate in an AdS background which is cutoff at some finite distance $u=u_{0}[8]$. To this we add an extra $\mathrm{U}(1)$ field, $V_{\mu}$ dual to the electromagnetic current $J_{\mu}^{\mathrm{EM}} \cdot{ }^{3}$ Recall that $u_{0}$ corresponds to the introduction of the QCD-scale in the field theory:

\footnotetext{
${ }^{2}$ We will keep to Lorentzian signature throughout since we seek information regarding the response of the thermal ensemble to small perturbations. This requires the use of real-time Green's functions [16].

${ }^{3}$ In chiral pertubation theory this $\mathrm{U}(1)$ external field is a subgroup of the local $\mathrm{SU}(3)_{L} \times \mathrm{SU}(3)_{R}[17]$.
} 
it enforces the mass-gap by hand by explicitly cutting-off any dynamics in the IR. For the reasons we explained in the introduction, here we are going to use a soft wall cut-off $[9,10]$. Formally we can introduce this cut-off by modifying the AdS bulk action to (we give only the term relevant for calculating the photon production rate)

$$
S \sim \int d^{5} x \sqrt{g}\left(-\frac{1}{4} F_{A B} F^{A B}+\cdots\right) \Rightarrow S \sim \int d^{5} x \sqrt{g} e^{-\Phi}\left(-\frac{1}{4} F_{A B} F^{A B}+\cdots\right)
$$

Here $A, B=t, x, y, z, u$ and the "dilaton" takes the fixed form $\Phi=c u$ where $c=\frac{\Lambda_{I R}^{2}}{(\pi T)^{2}}$, with $\Lambda_{I R}$ the IR scale below which physics is cut-off. This introduction into the action is formal in the sense that (1) we shall not consider $\Phi$ a dynamical field and (2) we assume that the presence of the cut-off does not affect the geometric AdS background, see also [10]. We thus still work with the metric (2.7) for the finite temperature version of AdS/QCD, but with the equation of motion for the fluctuations derived from action (2.8). We will discuss the validity of this approach in detail in section 4 .

For photon production, we need only the $\mathrm{U}(1)$ gauge field equation of motion $\partial_{A}\left(\sqrt{g} e^{-c u} g^{A B} g^{C D} F_{B D}\right)=0$ with $F_{A B}=\partial_{A} V_{B}-\partial_{B} V_{A}$ the Maxwell field strength. The 4 d electric fields are $E_{i} \equiv F_{t i}$ with $i=x, y, z$. Note that we use $A$ as a vector index and $V_{B}$ for the AdS gauge field. To compute the AdS boundary 2-point correlation function from which to extract the spectral density $\chi_{\mu \nu}$, we follow [11] and split the equation of motion into parts perpendicular $\left(V_{x}, V_{y} \equiv V_{\perp}\right)$ and parallel $\left(V_{z} \equiv V_{\|}\right)$to a predefined spatial threemomentum $\vec{k}=(0,0, k)$, the Gauss constraint ( $V_{0}$ e.o.m.) and the radial AdS $\left(V_{u}\right)$ equation of motion. After a Fourier transformation along $t, x, y, z$, and defining $\omega=\frac{k^{0}}{2 \pi T}, q=\frac{k}{2 \pi T}$, we find respectively

$$
\begin{array}{r}
\partial_{u}^{2} V_{\perp}+\left(\frac{\partial_{u} f}{f}-c\right) \partial_{u} V_{\perp}+\frac{\omega^{2}-q^{2} f}{u f^{2}} V_{\perp}=0 \\
\frac{q}{u f}\left(q V_{t}+\omega V_{\|}\right)-\left(\partial_{u}^{2} V_{t}+i(2 \pi T) \omega \partial_{u} V_{u}\right)+c\left(\partial_{u} V_{t}+i(2 \pi T) \omega V_{u}\right)=0 \\
\frac{\omega}{u f^{2}}\left(q V_{t}+\omega V_{\|}\right)+\left[\left(\frac{\partial_{u} f}{f}-c\right) \partial_{u} V_{\|}+\partial_{u}^{2} V_{\|}\right]-i(2 \pi T) q\left[\left(\frac{\partial_{u} f}{f}-c\right) V_{u}+\partial_{u} V_{u}\right]=0 .
\end{array}
$$

The equation of motion for $V_{u}$,

$$
\sqrt{g} e^{-c u} g^{u u}\left(g^{t t} \partial_{t} F_{t u}+g^{\|\|} \partial_{\|} F_{\| u}\right)=0
$$

can be simplified to

$$
V_{u}=\frac{i}{2 \pi T} \frac{\left(\omega \partial_{u} V_{t}+q f \partial_{u} V_{\|}\right)}{\left(\omega^{2}-q^{2} f\right)}
$$

Let us define $E_{\perp}=\omega V_{\perp}$ and $E_{\|}=q V_{t}+\omega V_{\|}$. From eq. (2.9) and combining eq. (2.11) 
with eq. (2.10) and eq. (2.13) in the gauge $V_{u}=0$ we obtain the two decoupled equations

$$
\begin{array}{r}
\partial_{u}^{2} E_{\perp}+\left(\frac{\partial_{u} f}{f}-c\right) \partial_{u} E_{\perp}+\frac{\omega^{2}-q^{2} f}{u f^{2}} E_{\perp}=0, \\
\partial_{u}^{2} E_{\|}+\left[\frac{\omega^{2} \partial_{u} f}{f\left(\omega^{2}-q^{2} f\right)}-c\right] \partial_{u} E_{\|}+\frac{\omega^{2}-q^{2} f}{u f^{2}} E_{\|}=0 .
\end{array}
$$

We shall need to solve these two equations to obtain the spectral density $\chi_{\mu \nu}$. These differential equations (2.14) and (2.15) have three regular singular points at $u= \pm 1,0$, and one irregular singular point at $\infty .^{4}$

Formal solutions for such equations are difficult to construct. Note that the irregular nature of the point at infinity becomes regular when we remove the IR-cutoff $c$. The irregular point, however, is outside the physical region of interest $u \in(0,1)$ and we can, for instance, solve the equations (2.9) and (2.15) near the boundary $u \rightarrow 0$ using Frobenius expansion $E=u^{\lambda} \sum_{n=0}^{\infty} a_{n} u^{n}$ where the indicial equation has solutions for $\lambda=0,1$.

To solve the equations $(2.14),(2.15)$ explicitly shall be the main part of this note. The solutions to these 5-d AdS equations of motion then give the 4-d field theory two point correlation as the functional derivative with respect to the boundary values of the on-shell AdS action

$$
S=-\left.\frac{1}{4 g_{B}^{2}} \int d^{4} x d u \sqrt{g} e^{-c u} F_{A B} F^{A B}\right|_{\text {on-shell }}
$$

with $g_{B}^{2}=16 \pi^{2} R / N_{c}^{2}$. Considering $V_{u}=0$ gauge, we can write this as

$$
\begin{aligned}
S_{\text {On-shell }} & =-\left.\frac{N_{c}^{2}}{32 \pi^{2} R} \int_{-\infty}^{\infty} d^{4} x\left(\sqrt{g} e^{-c u} V_{\mu} F^{u \mu}\right)\right|_{u=0} ^{u=1} \\
& =\left.\frac{N_{c}^{2} T^{2}}{16} \int_{-\infty}^{\infty} d^{4} x e^{-c u}\left(V_{t} \partial_{u} V_{t}-f V_{i} \partial_{u} V_{i}\right)\right|_{u=0} ^{u=1} .
\end{aligned}
$$

Fourier transforming to momentum space and selecting the particular direction chosen previously, we can rewrite the action using Minkowskian prescription formulated by Son and Starinets [16]. Together with the boundary condition that the solution of equations (2.9) and (2.15) must satisfy the incoming-wave boundary condition at the horizon $u=1$, the resulting on-shell action becomes

$$
\begin{aligned}
S_{\text {On-shell }}=\frac{N_{c}^{2} T^{2}}{16} \lim _{u \rightarrow 0} \int \frac{d \omega d q}{(2 \pi)^{2}} e^{-c u} & \\
& {\left[\frac{f}{q^{2} f-\omega^{2}} \partial_{u} E_{\|}(u, K) E_{\|}(u,-K)-\frac{f}{\omega^{2}} \partial_{u} E_{\perp}(u, K) E_{\perp}(u,-K)\right] . }
\end{aligned}
$$

\footnotetext{
${ }^{4}$ Recall that an irregular singular point for a differential equation $y^{\prime \prime}+P(x) y^{\prime}+Q(x) y=0$ is a point $x_{0}$ for which either $\lim _{x \rightarrow x_{0}}\left(x-x_{0}\right) P(x)$ or $\lim _{x \rightarrow x_{0}}\left(x-x_{0}\right)^{2} Q(x)$ diverges. The point at infinity is irregular if $\lim _{x \rightarrow \infty}(2-x P(x))$ or $\lim _{x \rightarrow \infty} x^{2} Q(x)$ diverges. Using that $f=\left(1-u^{2}\right)$ one clearly sees how the introduction of $c$ introduces a divergence in $\lim _{u \rightarrow \infty} 2-u\left(\partial_{u} \ln f-c\right)=\lim _{u \rightarrow \infty} 2+2 u^{2} /\left(1-u^{2}\right)+u c$.
} 
From eq. (2.18) and the condition described above, we can now compute the retarded current-current correlation function in term of two independent scalar functions ${ }^{5}$

$$
\begin{aligned}
& \Pi^{L}(K)=-\frac{N_{c}^{2} T^{2}}{8} \lim _{u \rightarrow 0} \frac{\partial_{u} E_{\|}(u, K)}{E_{\|}(u, K)}, \\
& \Pi^{T}(K)=-\frac{N_{c}^{2} T^{2}}{8} \lim _{u \rightarrow 0} \frac{\partial_{u} E_{\perp}(u, K)}{E_{\perp}(u, K)} .
\end{aligned}
$$

These functions in turn give us the photon and dilepton production at strong coupling via eq. (2.3) and eqs. (2.1) and (2.4).

\section{Solving the system}

In this section we will solve the equations (2.14) and (2.15) in order to compute the two scalar functions (2.19) and (2.20). Furthermore, we will take the imaginary part of those scalar functions and obtain the spectral density function (2.2) for finite temperature system.

The solutions which satisfy the incoming-wave boundary condition can be written in general as a Frobenius expansion near $u \rightarrow 1$

$$
E_{i}(u)=(1-u)^{-i \omega / 2} y_{i}(u),
$$

with $y_{i}(u)$ regular at $u=1$. We will solve and discuss these equations extensively for lightlike momenta relevant for photon-production, both semi-analytically for asymptotically small and large frequency and numerically for various values of the cut-off $c$ for the full range of momenta. For timelike and spacelike momenta we only present the numerical solution.

\subsection{Lightlike momenta}

As has been explained in section 2, the longitudinal part of the scalar functions vanishes for lightlike momenta and we just need to compute the transverse part.

\subsubsection{Analytic solutions for lightlike momenta at low and high frequency}

We are mainly interested in the effect of the IR-cut-off on photon production as compared to the previous AdS photon production calculation for scale-invariant $\mathcal{N}=4 \mathrm{SYM}$ [11]. In the low-frequency limit where its effect should be largest, we can solve (2.9) perturbatively using $\omega \ll 1$ as a small parameter. As noted in [11], there is a shortcut to do so. Given the two independent solutions $\phi_{1} \pm i \phi_{2}$ to the differential equation $\phi^{\prime \prime}+A(x) \phi^{\prime}+B(x) \phi=0$, the Wronskian times $\exp \left(\int^{x} A\left(x^{\prime}\right)\right)$ is strictly conserved

$$
\partial_{x}\left(e^{\int^{x} A\left(x^{\prime}\right)}\left[\bar{\phi} \partial_{x} \phi-\phi \partial_{x} \bar{\phi}\right]\right)=0 .
$$

The transverse scalar can be rewritten as

$$
\begin{aligned}
\Pi^{T}(K) & =\lim _{u \rightarrow 0} \Pi^{T}(u, K), \\
\Pi^{T}(u, K) & \equiv-\frac{N_{c}^{2} T^{2}}{8}\left[e^{-c u}\left(1-u^{2}\right) \frac{\bar{E}_{\perp}(u, K)}{\bar{E}_{\perp}(0, K)} \partial_{u} \frac{E_{\perp}(u, K)}{E_{\perp}(0, K)}\right] .
\end{aligned}
$$

\footnotetext{
${ }^{5}$ For a more detailed derivation of these functions see [18].
} 
The imaginary part of the transverse scalar $\Pi^{T}(u, K)$ is then propotional to the conserved Wronskian and therefore independent of the radial coordinate $u$ :

$$
\partial_{u} \operatorname{Im}\left[\Pi^{T}(u, K)\right]=0 .
$$

With this fact, we can evaluate the imaginary part of (3.3) at any given value of $u$ which is convenient to our calculation. Let us choose $u=1$. Because the transverse scalar (3.3) contains an explicit factor of $(1-u)$, only the pole in $\bar{E}_{\perp} \partial_{u} E_{\perp}$ will contribute. Recalling that for any finite frequency $\omega$ the boundary conditions determine $E_{\perp}(u)$ to be of the form (3.1), we immediately see that the undetermined regular part $y$ contains no pole by definition. Therefore without needing to solve the equation motion we see that

$$
\Pi^{T}(1, K)=\frac{-N_{c}^{2} T^{2}}{8}\left(\frac{-i \omega}{2}\right)\left[2 e^{-c}\left|\frac{y(1)}{y(0)}\right|^{2}\right] .
$$

The leading term in the limit $\omega \ll 1$ is the $\omega$-independent contribution to $|y(1) / y(0)|$. The determining equation (2.14) simplifies in that limit to effectively the first order equation (recall that $\omega=q$ for lightlike momenta)

$$
\partial_{u} \partial_{u} E_{\perp}+\left(\partial_{u}(\ln f-c u)\right) \partial_{u} E=0+\mathcal{O}\left(\omega^{2}\right) .
$$

The incoming wave boundary condition demands that the $\omega=0$ solution be regular at $u=1$. Since $f=(1-u)(1+u)$, this solution is the trivial constant one. Therefore

$$
\Pi^{T}(1, K)=\frac{i \omega N_{c}^{2} T^{2}}{8} e^{-c}+\mathcal{O}\left(\omega^{2}\right) .
$$

In appendix A we compute the same answer directly by solving the differential equation perturbatively in $\omega$, which shows explicitly that $E_{\perp}(u)=$ constant $+\mathcal{O}(\omega)$ is indeed the correct solution to the boundary conditions.

Given $\Pi(1, K)$, the trace of spectral density function at low-frequency limit for lightlike momenta in photon production is proportional to its $u$-independent imaginary part

$$
\begin{aligned}
\chi_{\mu}^{\mu}(\omega=q) & =-4 \operatorname{Im}\left(\Pi^{T}(\omega=q)\right) \\
& =\frac{\omega N_{c}^{2} T^{2}}{2} e^{-c}+\mathcal{O}\left(\omega^{2}\right) .
\end{aligned}
$$

For $c=0$, we reproduce back the result from [11] at the first order. The vanishing of $c$ corresponds to either the limit $T \rightarrow \infty$ or to removing the IR scale $\Lambda_{I R}$. We see explicitly our intuition confirmed that the trace of spectral density at low-frequency depends on the cutoff parameter $c$, while simultaneously reproducing the $\mathcal{N}=4$ result at high $T$.

At high-frequencies we do not expect the IR-cut-off to have a major effect. Let us show that to leading order the spectral function is in fact independent of the value of $c$ as one would expect. In this limit $\omega \gg 1$, the argument leading up to eq. (3.5) does not hold $^{6}$ and one cannot obtain the answer without solving the equation of motion (2.14).

\footnotetext{
${ }^{6}$ Note e.g. that in the singular term $(1-u)^{-i \omega / 2}$ the order of limits $u \rightarrow 1$ and $\omega \rightarrow \infty$ do not commute.
} 
Following [11], we will use the Langer-Olver method [19, 20] to find the solution. The first step is to redefine

$$
E_{\perp}(u)=\frac{e^{c u / 2}}{\sqrt{-f(u)}} y(u)
$$

for equation (2.14) and rewrite it as

$$
y^{\prime \prime}(x)=\left[\omega^{2} H(x)+G(x)\right] y(x),
$$

where $H(x)=\frac{x}{f(x)^{2}}$ and $G(x)=\frac{c^{2}}{4}-\frac{c x}{f(x)}-\frac{1}{f(x)^{2}}$ with $x=-u \in[-1,0]$. For large $\omega$ the first term on the r.h.s. dominates. Since it has a simple zero at $x=0$, we can transform eq. (3.10) to Airy's equation plus terms subleading in $\omega$. To do so, we introduce a new independent variable $\zeta$ and change variables to

$$
\zeta\left(\frac{d \zeta}{d x}\right)^{2}=H(x)=\frac{x}{\left(1-x^{2}\right)^{2}}
$$

Choosing conditions $\zeta(0)=0$ and $\zeta^{\prime}(0)>0$ determines $\zeta$ to be

$$
\zeta=\left[\frac{3}{2} \int_{0}^{x} \sqrt{H(t)} d t\right]^{2 / 3}
$$

Rescaling $y(x)$ to

$$
y=\left(\frac{d \zeta}{d x}\right)^{-1 / 2} W
$$

eq. (3.10) becomes

$$
\frac{d^{2} W}{d \zeta^{2}}=\left[\omega^{2} \zeta+\psi(\zeta)\right] W
$$

with

$$
\psi(\zeta)=\frac{5}{16 \zeta^{2}}+\frac{\left[4 H(x) H^{\prime \prime}(x)-5 H^{\prime 2}(x)\right]}{16 H^{3}(x)} \zeta+\frac{\zeta G(x)}{H(x)}
$$

For large $\omega$ we may ignore $\psi(\zeta)$ and the equation reduces to Airy's equation. To leading order the solution is thus

$$
W(\zeta)=A_{0} \operatorname{Ai}\left(\omega^{2 / 3} \zeta\right)+B_{0} \operatorname{Bi}\left(\omega^{2 / 3} \zeta\right)+\ldots,
$$

The incoming-wave boundary conditions at the horizon imply that $B_{0}$ should vanish. Thus the solution for $E_{\perp}(u)$ in asymptotic expansion for large $\omega$ is

$$
E_{\perp}(u)=\frac{A_{0} e^{c u / 2}}{\sqrt{-f(u)}}\left[\frac{-u}{f(u)^{2} \zeta(-u)}\right]^{-1 / 4} \operatorname{Ai}\left(\omega^{2 / 3} \zeta(-u)\right)+\ldots,
$$

and the transverse scalar at high-frequency limit equals

$$
\Pi^{T}=-\frac{N_{c}^{2} T^{2}}{8} \lim _{u \rightarrow 0}\left(\frac{c}{2}+\frac{1}{4} \partial_{u} \ln \left(\frac{-\zeta(-u)}{u}\right)+\frac{\partial_{u} \operatorname{Ai}\left(\omega^{2 / 3} \zeta(-u)\right)}{\operatorname{Ai}\left(\omega^{2 / 3} \zeta(-u)\right)}\right)+\ldots
$$


Before we move on, it is helpful to expand $\zeta(-u)$ around $u=0$

$$
\zeta(-u)=-(-1)^{2 / 3} u-\frac{2}{7}(-1)^{2 / 3} u^{3}+\mathcal{O}\left(u^{5}\right) .
$$

Therefore the middle term in (3.18),

$$
\partial_{u} \ln \left(\frac{-\zeta(-u)}{u}\right)=\frac{1}{(-1)^{2 / 3}+\ldots}\left(\frac{6}{7}(-1)^{2 / 3} u+\ldots\right),
$$

vanishes as $u \rightarrow 0$. Knowing the asymptotics of the Airy function the last term of (3.18) can be written as

$$
\begin{aligned}
\lim _{u \rightarrow 0} \frac{\left.\operatorname{Ai}^{\prime}\left(\omega^{2 / 3} \zeta(-u)\right)\right)}{\left.\operatorname{Ai}\left(\omega^{2 / 3} \zeta(-u)\right)\right)} & =-(-\omega)^{2 / 3} \frac{\operatorname{Ai}^{\prime}(0)}{\operatorname{Ai}(0)} \\
& =(-\omega)^{2 / 3} \frac{3^{1 / 3} \Gamma(2 / 3)}{\Gamma(1 / 3)}
\end{aligned}
$$

and thus we obtain

$$
\Pi^{T}=-\frac{N_{c}^{2} T^{2}}{8}\left(\frac{c}{2}+\frac{e^{2 \pi i / 3} \omega^{2 / 3} 3^{1 / 3} \Gamma(2 / 3)}{\Gamma(1 / 3)}\right) .
$$

Note that this transverse scalar therefore depends on $c$. However, only the real part does. The trace of the spectral density function in high-frequency limit for lightlike momenta

$$
\begin{aligned}
\chi_{\mu}^{\mu} & =-4 \operatorname{Im}\left(\Pi^{T}\right) \\
& \sim \frac{N_{c}^{2} T^{2}}{4} \frac{\omega^{2 / 3} 3^{5 / 6} \Gamma(2 / 3)}{\Gamma(1 / 3)} .
\end{aligned}
$$

does not depend on the cutoff parameter $c$ at least up to first order and yields the same result as the calculation in $\mathcal{N}=4 \mathrm{SYM}$. The fact that $c$ does appear in the real part of the transverse scalar indicates that at first subleading order the spectral density function will likely differ from the $\mathcal{N}=4$ result. The numerical results in the next section bear this out.

\subsubsection{Numerical solution for lightlike momenta}

The analytic asymptotic solutions are a guidance to the full spectral function. The full solutions of equation (2.9) for non-zero $c$ are very difficult to find, as we remarked earlier. This is due to the irregular singular point at $u=\infty$ for $c \neq 0$ where analytic solutions are not known. In this subsection we are going to look for numerical solutions for non-zero $c$.

We start from the general solution (3.1) which satisfies the incoming wave boundary condition. To set a parametrization of the initial conditions for the $u=1$ regular function $y_{i}(u)=E_{i}(1-u)^{i \omega / 2}$ of eq. (3.1), we write the general solution as a polynomial expansion around $u=1, y(u)=\sum_{n=0}^{\infty} a_{n}(1-u)^{n}$. Substituting (3.1) into equation (2.14) for lightlike momenta, we obtain the equation

$$
\begin{aligned}
& \sum_{n=0}^{\infty}\left[a_{n}\left(n-i \frac{\omega}{2}\right)^{2}(1-u)^{n-2}+c a_{n}\left(n-i \frac{\omega}{2}\right)(1-u)^{n-1}\right. \\
& \left.\quad-\sum_{m=0}^{\infty}\left[\frac{a_{n}}{2^{m+1}}\left(n-i \frac{\omega}{2}+\frac{\omega^{2}(m+1)}{4}\right)(1-u)^{n+m-1}-\frac{a_{n} \omega^{2}}{2^{m+2}}(1-u)^{n+m-2}\right]\right]=0 .
\end{aligned}
$$


The second sum (over $m$ ) arises from expanding $\frac{1}{1+u}=\sum_{n=0}^{\infty} \frac{1}{2^{n+1}}(1-u)^{n}$ and $\frac{1}{(1+u)^{2}}=$ $\sum_{n=0}^{\infty} \frac{(n+1)}{2^{n+2}}(1-u)^{n}$. In order to find the coefficients $a_{n}$, we have to solve this equation for each power of $(1-u)$ and obtain

$$
\begin{aligned}
(1-u)^{-2}: & a_{0} \text { (arbitrary) } \\
(1-u)^{-1}: & a_{1}=\frac{i \omega(c-1 / 2)}{2(1-i \omega)} a_{0} \\
\vdots & \\
(1-u)^{k-2}: & a_{k}=f_{k}(\omega, c) a_{0}
\end{aligned}
$$

with $f_{k}$ are functions of $\omega$ and $c$ which vanish at $\omega=0$. This gives us $y(u)$ and $y^{\prime}(u)$ at $u=1$ in terms of the above coefficients

$$
\begin{aligned}
y(1) & =a_{0}, \\
y^{\prime}(1) & =-a_{1}=-a_{0} \frac{i \omega(c-1 / 2)}{2(1-i \omega)} .
\end{aligned}
$$

These will be the two initial conditions for the differential equation for $y(u)$. The explicit differential equation it must satisfy is

$$
\begin{aligned}
y^{\prime \prime}+ & \left(\frac{i \omega}{1-u}-\frac{2 u}{1-u^{2}}-c\right) y^{\prime} \\
& +\left[\frac{\omega^{2} u}{\left(1-u^{2}\right)^{2}}+\frac{2 i \omega-\omega^{2}}{4(1-u)^{2}}-\frac{i \omega}{2(1-u)}\left(\frac{2 u}{1-u^{2}}+c\right)\right] y=0 .
\end{aligned}
$$

Notice that the initial conditions for $y(u)$ still depend on an arbitrary constant $a_{0}$. Physical quantities, such as the spectral density function, depend on ratios of $y(u)$ and its derivatives and are independent of this constant. We are therefore free to set it to any value; we will choose $a_{0}=1$.

Let us express the trace of spectral density function in terms of $y(u)$ :

$$
\chi_{\mu}^{\mu}=\frac{N_{c}^{2} T^{2}}{2}\left(\frac{\omega}{2}+\operatorname{Im}\left(\frac{y^{\prime}(0)}{y(0)}\right)\right) .
$$

Alternately we could use the modified Wronskian formulation for $\Pi^{T}((K)$, eq. (3.3) and evaluate it at $u=1$. An equivalent expression for the trace of spectral density function in this limit becomes

$$
\chi_{\mu}^{\mu}=\frac{\omega N_{c}^{2} T^{2}}{2} e^{-c} \frac{|y(1)|^{2}}{|y(0)|^{2}} .
$$

\subsubsection{The spectral density for lightlike momenta}

Solving eq. (3.27) numerically with initial conditions (3.26), we find the spectral density function $\chi_{\mu}^{\mu}$ for lightlike momenta for various values of the IR-cut-off $c .^{7}$ The results are shown in figure 1 and we clearly see the dependency at low frequencies on the IR-cut-off. The behaviour at high-frequency on the other hand appears less and less sensitive.

\footnotetext{
${ }^{7}$ Numerical solutions were obtained using the NDSolve routine in Mathematica.
} 


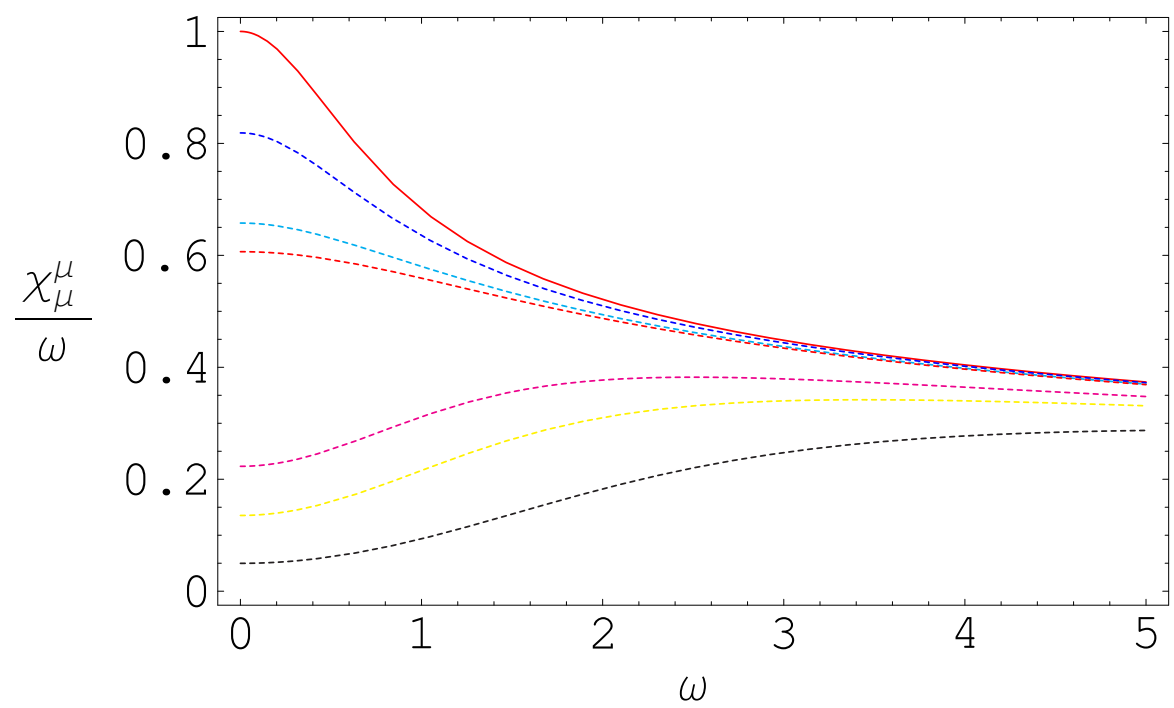

Figure 1. Trace of the spectral function for lightlike momenta in units of $\frac{1}{2} N_{c}^{2} T^{2}$, plotted as a function of frequency with $\omega \equiv k^{0} /(2 \pi T)$. The solid line (red) shows the exact result for $c=0$ and the dashed lines downward show numerical analysis for $c=0.2,0.419035,0.5,0.6,1.5,2,3$.

What is remarkable is the similarity between this soft-wall AdS/QCD result, Fig 1, for the trace of the spectral function for light-like momenta and of Mateos and Patiño for massive flavor deformations of the AdS dual of $\mathcal{N}=2$ theories, figure 3 in $[12,13]$. As we will discuss in section 4 , this similarity can be explained by relating the two computations. Inherently this then partially validates the soft-wall AdS/QCD model.

There is, however, one fundamental difference between the result here and the massive $\mathcal{N}=2$ computation. Both models are thermodynamically unstable for large IR-cut-off, signalling the transition back to the confining regime. In the $\mathcal{N}=2$ model this is clearly illustrated by the appearance of thermal resonances in the spectral function when formally evaluated beyond the critical cut-off. Fig1. shows that in AdS/QCD these resonances remain absent beyond the critical value $c>0.419035$ [10]. The absence of thermal resonances was presaged by Huot et al. [11]. Realizing that their results for photon production in the AdS dual of pure $\mathcal{N}=4 \mathrm{SYM}$ are unaffected by a hard-wall IR-cut-off, they speculated that this would be generic. It was premised on the fact that in the hard-wall case, the IR-cut-off is always inside the horizon. Rough dimensional analysis illustrates that the soft-wall case is similar: at the transition the cut-off scale $c^{-1} \simeq 2.5$ is beyond the horizon $u=1$. However, a similar argument holds for the massive $\mathcal{N}=2$ AdS dual. As we discuss in section 4 , the real reason for the absence of thermal resonances is probably simply that an blunt soft- or hard- IR-cut-off is too crude to capture this information.

\subsection{Timelike and spacelike momenta}

For time and space-like momenta, both $\Pi^{T}$ and $\Pi^{L}$ can contribute to spectral density function $\chi_{\mu}^{\mu}(K)$. Also for these cases, the mode equations (2.9) and (2.15) cannot be solved analytically for arbitrary frequency $(\omega)$ and wave vector $(q)$, and we determine the spectral function numerically. 


\subsubsection{Numerical solution for transverse scalar function}

Following the same procedure in numerical analysis for lightlike momenta above, we substitute the general solution (3.1) for transverse direction into (2.9) and obtain an equation for $y(u)$

$$
\begin{aligned}
y_{\perp}^{\prime \prime}+ & \left(\frac{i \omega}{1-u}-\frac{2 u}{1-u^{2}}-c\right) y_{\perp}^{\prime} \\
& +\left[\frac{\omega^{2}-q^{2}\left(1-u^{2}\right)}{u\left(1-u^{2}\right)^{2}}+\frac{2 i \omega-\omega^{2}}{4(1-u)^{2}}-\frac{i \omega}{2(1-u)}\left(\frac{2 u}{1-u^{2}}+c\right)\right] y_{\perp}=0 .
\end{aligned}
$$

As in the lightlike case, to determine the initial conditions we expand $y(u)=\sum_{n=0}^{\infty} a_{n}(1-$ $u)^{n}$ around $u=1$, with

$$
a_{0} \text { (arbitrary), } \quad a_{1}=\frac{\omega^{2}-q^{2}+i \omega(1 / 2-c)}{2(i \omega-1)} a_{0}, \quad a_{k}=f_{k}(\omega, q, c) a_{0}
$$

where again $f_{k}$ are functions of $\omega, q$ and $c$ which vanish at $\omega=q=0$. Using the modified Wronskian extension the imaginary part of transverse scalar function is therefore given by

$$
\operatorname{Im}\left(\Pi^{T}(K)\right)=-\frac{\omega N_{c}^{2} T^{2}}{8} e^{-c} \frac{\left|y_{\perp}(1)\right|^{2}}{\left|y_{\perp}(0)\right|^{2}}
$$

with $y(u)$ a solution to eq. (3.30) with initial conditions determined from eq. (3.31).

\subsubsection{Numerical solution for longitudinal scalar function}

Substitute (3.1) into the equation of motion for the longitudinal direction (2.15), we obtain

$$
\begin{aligned}
y_{\|}^{\prime \prime}+\left(\frac{i \omega}{1-u}-\frac{2 u \omega^{2}}{\left(1-u^{2}\right)\left(\omega^{2}-q^{2}\left(1-u^{2}\right)\right)}-c\right) y_{\|}^{\prime}+\left[\frac{\omega^{2}-q^{2}\left(1-u^{2}\right)}{u\left(1-u^{2}\right)^{2}}\right. \\
\left.+\frac{2 i \omega-\omega^{2}}{4(1-u)^{2}}-\frac{i \omega}{2(1-u)}\left(\frac{2 u \omega^{2}}{\left(1-u^{2}\right)\left(\omega^{2}-q^{2}\left(1-u^{2}\right)\right)}+c\right)\right] y_{\|}=0 .
\end{aligned}
$$

Expanding $y_{\|}(u)=\sum_{n=0}^{\infty} a_{n}(1-u)^{n}$ around $u=1$ gives us

$$
a_{0} \text { (arbitrary), } \quad a_{1}=\frac{\omega^{2}-q^{2}+i \omega\left(\frac{1}{2}-c-\frac{2 q^{2}}{\omega^{2}}\right)}{2(i \omega-1)} a_{0}, \quad a_{k}=f_{k}(\omega, q, c) a_{0},
$$

where again $f_{k}$ are functions of $\omega, q$ and $c$ which vanish at $\omega=q=0$. The imaginary part of the longitudinal scalar function is

$$
\operatorname{Im}\left(\Pi^{L}(K)\right)=-\frac{\omega N_{c}^{2} T^{2}}{8}\left(\frac{1}{2}+\operatorname{Im}\left(\frac{y_{\|}^{\prime}(0)}{\omega y_{\|}(0)}\right)\right)
$$

with $y_{\|}(u)$ the solution to (3.33) with initial conditions determined from eq. (3.34). 


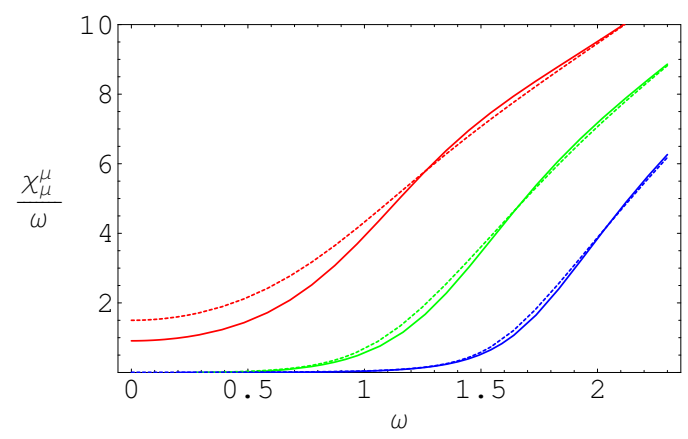

Figure 2. Spectral function trace $\chi_{\mu}^{\mu} / \omega$, in units of $N_{c}^{2} T^{2} / 2$, plotted as a function of $\omega$. The solid lines describe $c=0.5$ and the dashed lines for $c=0$ while different colors represent $q=0$ (red), $q=1$ (green), and $q=1.5$ (blue).

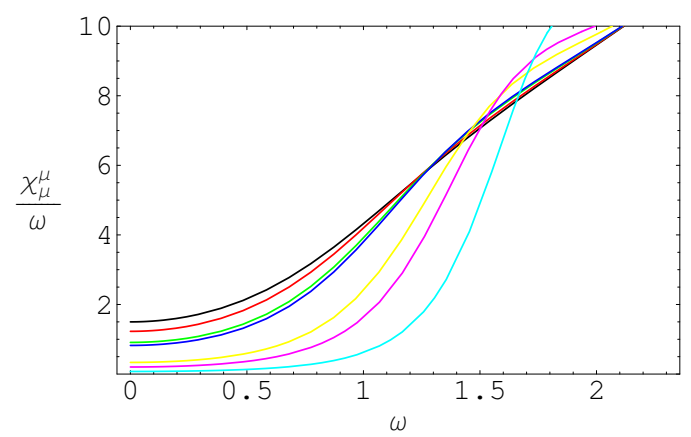

Figure 3. Spectral function trace $\chi_{\mu}^{\mu} / \omega$, in units of $N_{c}^{2} T^{2} / 2$, plotted as a function of $\omega$ for $q=0$ and various values of $c=0$ (black), $c=0.2$ (red), $c=0.5$ (green), $c=0.6$ (bue), $c=1.5$ (yellow), $c=2$ (magenta), and $c=3($ cyan).

\subsubsection{The spectral density for time- and space-like momenta}

Following formula (2.3), we can now write the trace of spectral function for time- and space-like momenta as

$$
\chi_{\mu}^{\mu}(K)=\frac{\omega N_{c}^{2} T^{2}}{2}\left[e^{-c} \frac{\left|y_{\perp}(1)\right|^{2}}{\left|y_{\perp}(0)\right|^{2}}+\frac{1}{4}+\frac{1}{2 \omega} \operatorname{Im}\left(\frac{y_{\|}^{\prime}(0)}{y_{\|}(0)}\right)\right] .
$$

The complete results for $\chi_{\mu}^{\mu}$ are plotted in figure 2 and figure 3 as a function of frequency for several values of the spatial momentum. As we increase the value for $c$, one clearly sees that at low momenta the function decreases compared to $c=0$.

\subsection{Electrical conductivity}

With the spectral density in hand, it is now straightforward to compute the electrical conductivity $\sigma$. Here, we will use eq. (2.6) as we have an analytic expression of the spectral 
density for lightlike momenta. Substituting (3.8) into (2.6) yields

$$
\begin{aligned}
\sigma & =\lim _{k^{0} \rightarrow 0} \frac{e^{2}}{4 T} \frac{\chi_{\mu}^{\mu}(\omega=q)}{e^{k^{0} / T}-1} \\
& =\lim _{k^{0} \rightarrow 0} \frac{e^{2}}{8 \pi} \frac{N_{c}^{2} \exp (-c) k^{0}\left(1+O\left(k^{0}\right)\right)}{k^{0} / T\left(1+O\left(k^{0}\right)\right)} \\
& =e^{2} \frac{N_{c}^{2} T}{16 \pi} \exp (-c),
\end{aligned}
$$

with $e$ the electric charge. We again note the presence of the scaling factor $e^{-c}$ which dampens the IR-properties, including charge diffusion, of the system. Note in particular that this IR-suppresion is also present in the charge susceptibility $\Xi=N_{c}^{2} T^{2} c / 8\left(e^{c}-1\right)$ and the more "universal" diffusion constant $D \equiv \sigma / e^{2} \Xi=\left(1-e^{-c}\right) / 2 \pi T c$ (see appendix B). Physically this makes sense, as a mass-gap should dampen any hydrodynamic behaviour and the general AdS/CFT computation for scale-dependent currents

$$
S_{\mathrm{AdS}} \sim \int d^{4} x d u \sqrt{-g} \frac{1}{g_{\mathrm{eff}}^{2}(u)} F_{A B} F^{A B},
$$

demonstrates this explicitly $[21]^{8}$

$$
D \sim \frac{1}{g_{\mathrm{eff}}^{2}(u=1)} \int_{0}^{1} d u g_{\mathrm{eff}}^{2}(u) \ldots
$$

\section{Conclusion: soft wall cut-offs as an IR mass-gap}

The essential new ingredient in Soft-wall AdS/QCD is the ad-hoc cut-off of the radial AdS-direction. It is intended to capture the dominant effects of the scale dependence of QCD [8, 10]. However, its ad-hoc introduction opens it to criticism; especially when interpreted as a dilaton-profile without taking into account back-reaction effects or the dilaton equation of motion (see the footnote in the introduction). On the other hand the succesful results of the model $[9,10]$, suggest that it does capture the essential IR behaviour correctly.

The result for AdS/QCD photon production supports this further. As previously emphasized it closely resembles photon production due to quarks for $\mathcal{N}=2$ theories with massive flavor in the probe approximation $N_{f} \ll N_{c}[12,13]$. These theories descend from brane-constructions in string theory, and therefore have no ad hoc component to criticise. Recall that in these theories, the probe approximation means that one may consider the flavor group as a global symmetry. The U(1) theory with respect to which photons are defined is a subgroup of this group and the tunable quark mass - a free parameter in the brane construction - functions as the scale in these theories. On the other hand, because the matter and symmetry content is different from QCD, one could question how relevant massive $\mathcal{N}=2$ SQCD results are to reality. The observation we make now is that the

\footnotetext{
${ }^{8}$ This suggests a trivial violation of the PSS shear-viscosity-bound by IR-suppressing hydrodynamic behaviour. As the derivation of the viscosity in [21] suggests, however, and the explicit computation in massive $\mathcal{N}=2$ models shows [22], this is not case.
} 
resemblence between the trace of the spectral function $\chi_{\mu}^{\mu}$ in these $\mathcal{N}=2$ SQCD theories as a function of the quark mass $m$ and the AdS/QCD spectral function as a function of the IR-cut-off $c$ can be mathematically explained. Both therefore demonstrate again that AdS/CFT results are remarkable universal and robust across fundamentally different theories. This is therefore strong support for soft-wall AdS/QCD, despite its ad-hoc IR-cutoff, as well as massive $\mathcal{N}=2 \mathrm{SQCD}$, despite its unrealistic matter content, as descriptions of QCD.

To relate the massive $\mathcal{N}=2 \mathrm{SQCD}$ result to AdS/QCD, we note that Mateos and Patiño showed that in $\mathcal{N}=2$ SQCD the defining equation relevant for the trace of the spectral function for lightlike momenta can be deduced from an action ${ }^{9}$

$$
S \sim \int d u d x_{0} d x_{1}\left[-P(u)\left(\partial_{0} V_{\perp}\right)^{2}+f P(u)\left(\partial_{1} V_{\perp}\right)^{2}+Q(u)\left(\partial_{u} V_{\perp}\right)^{2}\right],
$$

where

$$
\begin{aligned}
P(u) & =\frac{u^{3} \sqrt{g\left(\psi_{m, 0}(u), u\right)}}{u f}, \\
Q(u) & =f \frac{\left(1-\psi_{m, 0}^{2}(u)\right)^{3}}{u^{3} \sqrt{g\left(\psi_{m, 0}(u), u\right)}} \\
& =\frac{1}{u} \frac{u^{3} f \sqrt{g\left(\psi_{m, 0}(u), u\right)}}{u f} \frac{u^{2} f^{2}\left(1-\psi_{m, 0}^{2}(u)\right)^{3}}{u^{6} g\left(\psi_{m, 0}(u), u\right)} .
\end{aligned}
$$

Here $f=f(u)=\left(1-u^{2}\right)$ is the non-extremality function in the D3-brane metric (2.7). The function $\psi_{m, 0}(u)$ is the solution to the embedding equation of motion for the D7-flavor brane derived from the DBI-action

$$
S \sim \int d u \sqrt{g\left(\psi_{m}(u), u\right)}=\int d u \frac{1}{u^{3}}\left(1-\psi_{m}^{2}\right) \sqrt{1-\psi^{2}+4 u^{2} f \psi^{\prime 2}},
$$

i.e. $g(\psi(u), u)$ is the induced metric on the flavor brane. The $u=0$ boundary behavior of the solution $\psi_{m, 0}=\frac{m}{\sqrt{2}} u^{1 / 2}+\Lambda u^{3 / 2}+\ldots$ is determined by the masses $m$ and condensate expectation value $\langle q q\rangle \sim \Lambda$ of the quarks. For the massless theory $\psi_{m=0,0}=0$ and $\sqrt{g}=$ $u^{-3}$. Thus to find the spectral function, one must first solve the differential equation for $\psi_{m}(u)$ with the appropriate boundary conditions and then solve the differential equation for $V_{\perp}[12,13]$. The first step correctly incorporates the backreaction of the modified IR-physics as opposed to the AdS/QCD ad-hoc cut-off.

The massive case $\psi_{m, 0}(u) \neq 0$ is therefore a step more involved than the massless case, unlike AdS/QCD where the scale is a mild modification $c \neq 0$ of the defining differential equation (2.14). However, searching for a closer match, one quickly realizes that the massless equation (for lightlike momenta $\omega=\vec{k}$ ),

$$
\begin{aligned}
\partial_{u}^{2} V_{\perp}+\partial_{u}(\ln Q) \partial_{u} V_{\perp}+\vec{k}^{2}(1-f) \frac{P}{Q} V_{\perp} & =0 \\
\Rightarrow \partial_{u}^{2} V_{\perp}+\partial_{u}(\ln (f)) \partial_{u} V_{\perp}+\vec{k}^{2}(1-f) \frac{(u f)^{-1}}{f} V_{\perp} & =0,
\end{aligned}
$$

\footnotetext{
${ }^{9}$ We only consider the D3/D7 brane set-up of $[12,13]$. The gauge/gravity duality for the D4/D6 brane set-up they also consider is not yet fully understood.
} 
is exactly the AdS/QCD equation (2.14) for $c=0$ and we are therefore lead to consider a change of variables for the massive case that resembles that of the massless case. Thus we define a new variable $\tilde{u}$ such that

$$
d u \frac{u^{3} \sqrt{\left.g\left(\psi_{m, 0}(u), u\right)\right)}}{u f}=d \tilde{u} \frac{1}{\tilde{u} \tilde{f}}
$$

with $\tilde{f} \equiv f(\tilde{u})$. By construction the parameter $P$ in the new variable is identical to the massless case and $Q$ is seen to be a mild modification

$$
\begin{aligned}
& P(\tilde{u})=\frac{1}{\tilde{u} f(\tilde{u})}, \\
& Q(\tilde{u})=f(\tilde{u}) \frac{\tilde{u}\left(1-\psi_{m, 0}^{2}\right)^{3}}{u(\tilde{u})} .
\end{aligned}
$$

Note that the solution to the massive embedding equation of motion, $\psi_{m, 0} \neq 0$, is implicit in the transformation (4.5). In this new variable, however, we see, that its specific form only mildly modifies the massless differential equation

$$
\partial_{\tilde{u}}^{2} V_{\perp}+\partial_{\tilde{u}}\left[\ln (\tilde{f})+\ln \left(\left(1-\psi_{m, 0}^{2}\right)^{3} \frac{\tilde{u}}{u(\tilde{u})}\right)\right] \partial_{\tilde{u}} V_{\perp}+\vec{k}^{2}(1-\tilde{f}) \frac{(\tilde{u} \tilde{f})^{-1}}{f\left(1-\psi^{2}\right)^{3}} V_{\perp}=0 .
$$

and the close relation to AdS/QCD is now apparent. The resemblance of the spectral functions is especially explained, if we recall that it is primarily determined by the $u=0$ behaviour of the solution (2.20). ${ }^{10}$ As we know what the $u=0$ behaviour of the solution $\psi_{m, 0}=\frac{m}{\sqrt{2}} u^{1 / 2}+\ldots$ must be, eq. (4.5) shows that asymptotically $\tilde{u}=u+\frac{m^{2}}{4} u^{2}+\ldots$ and we can putatively identify the mass $m$ with the IR-cut-off $c$ :

$$
\begin{aligned}
-c \tilde{u} & \simeq \ln \left(1-\psi_{m, 0}^{2}\right)^{3} \frac{\tilde{u}}{u}=\ln \left(1-\frac{m^{2}}{2} \tilde{u}+\ldots\right)^{3}-\ln \left(1-\frac{m^{2}}{4} \tilde{u}+\ldots\right) \\
& \simeq-\frac{5}{4} m^{2} \tilde{u}+\ldots
\end{aligned}
$$

The map between AdS/QCD and $\mathcal{N}=2$ SQCD is not exact; clearly we should not have expected it to be. The latter shows thermal resonances in the spectral function for masses $m>1.3092$ which is the value beyond which the AdS black-hole solution becomes thermodynamically unstable $[12,13]$. The AdS/QCD description is much cruder as is no resonances show up even beyond the unstable regime $c>0.419035$. These thermal resonances are encoded in the subtleties of the embedding function $\psi_{m, 0}(u)$ which carries more information than just the mass as an IR-cut-off. Precisely, the embedding function determines whether the flavor D7-brane is in "Minkowski embedding" or "black hole embedding" corresponding to the low $T$ confining or high $T$ deconfining phase $[12,13]$. Clearly, the $\mathcal{N}=2$ SQCD theory has a more detailed description at the physics. On the other hand, the results here do show that in the stable phase the simple AdS/QCD model describes the IR-consequences of a mass-gap remarkably well and the above derivation explains mathematically why. This in itself lends support to continue to study AdS/QCD as a good toy model for real-world physics.

\footnotetext{
${ }^{10}$ One should be careful in that the change of coordinates (4.5) in principle will also change the boundary conditions one must impose.
} 


\section{Acknowledgments}

We are grateful to D. Mateos for correspondence. KS thanks and acknowledges the hospitality of the Galileo Galilei Institute in Firenze. This research was supported in part by a VIDI Innovative Research Incentive Grant from the Netherlands Organisation for Scientific Research (NWO).

\section{A Spectral function low frequency limit for lightlike momenta}

Here we find an analytic expression for the low-frequency limit of the transverse scalar and spectral density for lightlike momenta by solving the differential equation for the $E_{\perp}(u)$ perturbatively, rather than using the Wronskian shortcut, explained above eq. (3.2).

We first extract the other regular singularity at $u=-1$, writing

$$
E_{\perp}(u)=(1-u)^{-i \omega / 2}(1+u)^{-\omega / 2} Y(u)
$$

with $Y(u)$ regular at $u=1$ and substitute this into (2.9). Changing variables to $v=$ $1 / 2(1-u)$, we obtain the differential equation

$$
\begin{aligned}
v(1-v) Y^{\prime \prime}+\left[(1-i \omega)-(2-i \omega-\omega-2 c) v-2 c v^{2}\right] Y^{\prime} \\
-\left\{\frac{1}{2}\left[-\omega-i \omega+i \omega^{2}\right]-c[\omega v-i \omega+i \omega v]\right\} Y=0 .
\end{aligned}
$$

In the absence of the IR-cutoff, $c=0$, we recognize a hypergeometric equation with solution [11]

$$
Y(u)={ }_{2} F_{1}\left(1-\frac{1}{2}(1+i) \omega,-\frac{1}{2}(1+i) \omega ; 1-i \omega ; \frac{1}{2}(1-u)\right) .
$$

As we noted earlier, the presence of $c$ changes the nature of the equation and no formal solution is known. On physical grounds we expect the effects of $c$ to dominate the low frequency part of the spectral function. Expanding $Y(u)$ as

$$
Y=Y_{0}+\omega Y_{1}+\omega^{2} Y_{2}+\omega^{3} Y_{3}+\cdots
$$

we find to first order in $\omega$,

$$
\begin{aligned}
& \omega^{0}: \quad v(1-v) Y_{0}^{\prime \prime}+[1-2 v+2 c v(1-v)] Y_{0}^{\prime}=0, \\
& \omega^{1}: \quad v(1-v) Y_{1}^{\prime \prime}+[v-i(1-v)] Y_{0}^{\prime}+[1-2 v+2 c v(1-v)] Y_{1}^{\prime} \\
& +\left\{\frac{1}{2}(1+i)+c[v-i(1-v)]\right\} Y_{0}=0 .
\end{aligned}
$$

These two equations have solutions

$$
\begin{aligned}
Y_{0}(v)= & A+B\left[e^{-2 c} \operatorname{Ei}(2 c-2 c v)-\operatorname{Ei}(-2 c v)\right], \\
Y_{1}(v)= & C+\frac{A}{2}[\ln (v-1)+i \ln v]+ \\
& {\left[e^{-2 c} \operatorname{Ei}(2 c-2 c v)-\operatorname{Ei}(-2 c v)\right]\left[D+\frac{B}{2}[\ln (v-1)+i \ln v]\right], }
\end{aligned}
$$


with $A, B, C, D$ constants of integration and $\operatorname{Ei}(x)=-\int_{-x}^{\infty} \frac{e^{-t}}{t} d t$ the exponential integral function. To determine the integration constants, recall that by construction the solutions must be regular as $v \rightarrow 0(u \rightarrow 1)$. Since the exponential integral $\operatorname{Ei}(v)$ diverges at $v=0$, we must set $B=0$. To determine regularity of $Y_{1}(v)$, recall that $\operatorname{Ei}(x)$ can be written as

$$
\operatorname{Ei}(-x)=\gamma+\ln x+\sum_{n=1}^{\infty} \frac{(-1)^{n} x^{n}}{n ! n}, \quad \text { for } \quad x>0
$$

with $\gamma$ the Euler-Mascheroni constant. Since the variable $v \in[0,1 / 2]$, and $c>0$, regularity at $v=0$ demands $D=i A / 2$. For convenience, let us also redefine the constant $C=i \tilde{C} A / 2$. Substituting those constants into $Y_{1}$, we obtain the solution for $E_{\perp}$ in the low frequency limit

$$
\begin{aligned}
E_{\perp}(u)=A(1-u)^{-i \omega / 2}(1+u)^{-\omega / 2}\{ & 1+i \frac{\omega}{2}\left[\tilde{C}+e^{-2 c} \operatorname{Ei}(c(1+u))-\operatorname{Ei}(c(u-1))\right. \\
& \left.\left.-i \ln \left(\frac{u+1}{2}\right)+\ln \left(\frac{1-u}{2}\right)\right]+\mathcal{O}\left(\omega^{2}\right)\right\} .
\end{aligned}
$$

Using the definition of the exponential integral function, we straightforwardly obtain the leading low-frequency contribution to transverse scalar function

$$
\begin{aligned}
\Pi^{T}(\omega=q) & =-\frac{N_{c}^{2} T^{2}}{8}\left[-i \frac{\omega}{2}-\frac{\omega}{2}+\frac{i \omega}{2}\left(c e^{-2 c} \operatorname{Ei}^{\prime}(c)-c \operatorname{Ei}^{\prime}(-c)-i-1\right)+\mathcal{O}\left(\omega^{2}\right)\right] \\
& =\frac{i \omega N_{c}^{2} T^{2}}{16}\left[-2 i-\left(e^{-c}+e^{-c}\right)+\mathcal{O}\left(\omega^{2}\right)\right] .
\end{aligned}
$$

This is the exact answer. The imaginary part computed via the conserved Wronskian shortcut (3.7) clearly agrees.

\section{B The susceptibility and the diffusion constant}

We follow the procedure to compute the diffusion constant described in [7]. Using the gauge $V_{u}=0$, we can rewrite equation (2.10) as

$$
V_{\|}=\frac{u f}{q \omega} V_{t}^{\prime \prime}-c \frac{u f}{q \omega} V_{t}^{\prime}-\frac{q}{\omega} V_{t}
$$

Substituting into equation (2.13) we obtain a second order differential equation for $E=V_{t}^{\prime}$

$$
E^{\prime \prime}+\left[\frac{(u f)^{\prime}}{u f}-c\right] E^{\prime}+\left[\frac{\omega^{2}-q^{2} f}{u f^{2}}-c \frac{(u f)^{\prime}}{u f}\right] E=0 .
$$

Imposing the same incoming-wave boundary condition as before and extracting the singularity at the horizon $u=1$, we rewrite $E=(1-u)^{-i \omega / 2} y$, where $y$ is a regular function at the horizon. The function $y$ must obey the equation

$$
\begin{aligned}
& y^{\prime \prime}+\left[\frac{i \omega}{1-u}+\frac{(u f)^{\prime}}{u f}-c\right] y^{\prime}+ \\
& \quad+\left[\frac{i \omega(i \omega+2)}{4(1-u)^{2}}+\frac{i \omega\left((u f)^{\prime}-c u f\right)}{2 u f(1-u)}+\frac{\omega^{2}-q^{2} f}{u f^{2}}-c \frac{(u f)^{\prime}}{u f}\right] y=0 .
\end{aligned}
$$


For low frequency and momentum, we again solve the equation pertubatively in $\omega$ and $q$

$$
y(u)=y_{00}+\omega y_{10}+q^{2} y_{02}+\cdots .
$$

Up to first order in $\omega$ and $q^{2}$, we find the system of equations

$$
\begin{aligned}
& \omega^{0} q^{0}: y_{00}^{\prime \prime}+\left[\frac{(u f)^{\prime}}{u f}-c\right] y_{00}^{\prime}-c \frac{(u f)^{\prime}}{u f} y_{00}=0 \\
& \omega^{1} q^{0}: y_{10}^{\prime \prime}+\frac{i}{1-u} y_{00}^{\prime}+\left[\frac{(u f)^{\prime}}{u f}-c\right] y_{10}^{\prime}+\left[\frac{i}{2(1-u)^{2}}+\frac{i\left((u f)^{\prime}-c u f\right)}{2 u f(1-u)}\right] y_{00} \\
& \omega^{0} q^{2}:-c \frac{(u f)^{\prime}}{u f} y_{10}^{\prime \prime}+\left[\frac{(u f)^{\prime}}{u f}-c\right] y_{02}^{\prime}-c \frac{(u f)^{\prime}}{u f} y_{02}-\frac{f}{u f^{2}} y_{00}=0 .
\end{aligned}
$$

Using the same analysis for the low frequency of spectral function as described in the previous appendix, the solutions regular at $u=1$ are found to be

$$
\begin{aligned}
& y_{00}=A e^{c u} \\
& y_{10}=\frac{i A}{2} e^{c u+c}\left[C_{10}+2 \operatorname{Ei}(-c u)-e^{c} \operatorname{Ei}(-c(1+u))-e^{-c}(\operatorname{Ei}(c(1-u))-\ln (u-1))\right] \\
& y_{02}=\frac{A}{2 c} e^{c u+c}\left[C_{02}-2 \operatorname{Ei}(-c u)+e^{c} \operatorname{Ei}(-c(1+u))\right. \\
&\left.\quad+e^{-c}\left(\operatorname{Ei}(c(1-u))+2 \ln u-\ln \left(u^{2}-1\right)\right)\right]
\end{aligned}
$$

where $A$ and $C_{10}, C_{02}$ are constants independent of $u$. We can determine $A$ in terms of the boundary values of $V_{t}$ and $V_{\|}$at $u \rightarrow 0$ defined as

$$
\begin{aligned}
& \lim _{u \rightarrow 0} V_{t}(u)=V_{t}^{0}, \\
& \lim _{u \rightarrow 0} V_{\|}(u)=V_{\|}^{0} .
\end{aligned}
$$

Substituting the solution for $E=V_{t}^{\prime}$ into equation (B.1) and taking limit $u \rightarrow 0$, the integration constants $C_{10}, C_{02}$ drop out and we can determine $A$ to be

$$
A=\frac{q^{2} V_{t}^{0}+\omega q V_{\|}^{0}}{i \omega e^{c}-\frac{e^{c}}{c}\left(1-e^{-c}\right) q^{2}+O\left(\omega^{2}, \omega q^{2}, q^{4}\right)} .
$$

We recognize the hydrodynamic pole and as explained in [7] we can now compute the time-time component of the retarded thermal Green's function of two currents

$$
G_{t t}=\frac{N_{c}^{2} T^{2} q^{2} e^{-c}}{8\left(i \omega-\frac{\left(1-e^{-c}\right)}{c} q^{2}\right)}+\cdots
$$

Thus the time-time component of the spectral density function at low frequency and momentum equals

$$
\chi_{t t}\left(k^{0}, \vec{k}\right)=-2 \operatorname{Im}\left[G_{t t}\right]=\frac{N_{c}^{2} T k^{0}|\vec{k}|^{2} e^{-c}}{8 \pi\left(\left(k^{0}\right)^{2}+D|\vec{k}|^{2}\right)}+\ldots
$$


with $D=\frac{\left(1-e^{-c}\right)}{2 \pi T c}$ the diffusion constant. Comparing the result with the universal hydrodynamic behaviour

$$
\chi_{t t}\left(k^{0}, \vec{k}\right)=\frac{2 \omega D|\vec{k}|^{2}}{\left(k^{0}\right)^{2}+\left(D|\vec{k}|^{2}\right)^{2}} \Xi+\ldots
$$

the charge susceptibility $\Xi$ is seen to equal $\Xi=\frac{N_{c}^{2} T^{2} c}{8\left(e^{c}-1\right)}$ and naturally satisfies the Einstein relation $\Xi=\sigma / e^{2} D$.

Open Access. This article is distributed under the terms of the Creative Commons Attribution Noncommercial License which permits any noncommercial use, distribution, and reproduction in any medium, provided the original author(s) and source are credited.

\section{References}

[1] J.M. Maldacena, The large- $N$ limit of superconformal field theories and supergravity, Adv. Theor. Math. Phys. 2 (1998) 231 [Int. J. Theor. Phys. 38 (1999) 1113] [hep-th/9711200] [SPIRES].

[2] S.S. Gubser, I.R. Klebanov and A.M. Polyakov, Gauge theory correlators from non-critical string theory, Phys. Lett. B 428 (1998) 105 [hep-th/9802109] [SPIRES].

[3] E. Witten, Anti-de Sitter space and holography, Adv. Theor. Math. Phys. 2 (1998) 253 [hep-th/9802150] [SPIRES].

[4] S.S. Gubser, I.R. Klebanov and A.W. Peet, Entropy and temperature of black 3-branes, Phys. Rev. D 54 (1996) 3915 [hep-th/9602135] [SPIRES].

[5] I.R. Klebanov and A.A. Tseytlin, Entropy of near-extremal black p-branes, Nucl. Phys. B 475 (1996) 164 [hep-th/9604089] [SPIRES].

[6] E. Witten, Anti-de Sitter space, thermal phase transition and confinement in gauge theories, Adv. Theor. Math. Phys. 2 (1998) 505 [hep-th/9803131] [SPIRES].

[7] G. Policastro, D.T. Son and A.O. Starinets, From AdS/CFT correspondence to hydrodynamics, JHEP 09 (2002) 043 [hep-th/0205052] [SPIRES].

[8] J. Erlich, E. Katz, D.T. Son and M.A. Stephanov, QCD and a holographic model of hadrons, Phys. Rev. Lett. 95 (2005) 261602 [hep-ph/0501128] [SPIRES].

[9] A. Karch, E. Katz, D.T. Son and M.A. Stephanov, Linear confinement and AdS/QCD, Phys. Rev. D 74 (2006) 015005 [hep-ph/0602229] [SPIRES].

[10] C.P. Herzog, A holographic prediction of the deconfinement temperature, Phys. Rev. Lett. 98 (2007) 091601 [hep-th/0608151] [SPIRES].

[11] S. Caron-Huot, P. Kovtun, G.D. Moore, A. Starinets and L.G. Yaffe, Photon and dilepton production in supersymmetric Yang-Mills plasma, JHEP 12 (2006) 015 [hep-th/0607237] [SPIRES].

[12] D. Mateos, String theory and RHIC physics: the fundamental story, talk at the Strings 2007, June 25-29, Madrid, Spain (2007).

[13] D. Mateos and L. Patino, Bright branes for strongly coupled plasmas, JHEP 11 (2007) 025 [arXiv:0709.2168] [SPIRES]. 
[14] P. Stankus, Direct photon production in relativistic heavy-ion collisions, Ann. Rev. Nucl. Part. Sci. 55 (2005) 517 [SPIRES].

[15] M. Le Bellac, Thermal field theory, Cambridge University Press, Cambridge U.K.(1996).

[16] D.T. Son and A.O. Starinets, Minkowski-space correlators in AdS/CFT correspondence: recipe and applications, JHEP 09 (2002) 042 [hep-th/0205051] [SPIRES].

[17] S. Scherer and M.R. Schindler, A chiral perturbation theory primer, hep-ph/0505265 [SPIRES].

[18] P.K. Kovtun and A.O. Starinets, Quasinormal modes and holography, Phys. Rev. D 72 (2005) 086009 [hep-th/0506184] [SPIRES].

[19] F.W.J. Olver, The asymptotic solution of linear differential equations of second order fro large values of a parameter, Phil. Trans. Roy. Soc. Lon. Ser. A 247 (1954) 307.

[20] F.W.J. Olver, Asymptotics and special functions, A.K. Peters, Wellesley U.S.A. (1997).

[21] P. Kovtun, D.T. Son and A.O. Starinets, Holography and hydrodynamics: diffusion on stretched horizons, JHEP 10 (2003) 064 [hep-th/0309213] [SPIRES].

[22] D. Mateos, R.C. Myers and R.M. Thomson, Holographic viscosity of fundamental matter, Phys. Rev. Lett. 98 (2007) 101601 [hep-th/0610184] [SPIRES].

[23] U. Gürsoy, E. Kiritsis, L. Mazzanti and F. Nitti, Deconfinement and gluon plasma dynamics in improved holographic QCD, Phys. Rev. Lett. 101 (2008) 181601 [arXiv:0804.0899] [SPIRES].

[24] U. Gürsoy, E. Kiritsis, L. Mazzanti and F. Nitti, Holography and thermodynamics of $5 D$ dilaton-gravity, JHEP 05 (2009) 033 [arXiv:0812.0792] [SPIRES].

[25] U. Gürsoy, E. Kiritsis, L. Mazzanti and F. Nitti, Improved holographic yang-mills at finite temperature: comparison with data, Nucl. Phys. B 820 (2009) 148 [arXiv:0903.2859] [SPIRES].

[26] S.S. Gubser and A. Nellore, Mimicking the QCD equation of state with a dual black hole, Phys. Rev. D 78 (2008) 086007 [arXiv:0804.0434] [SPIRES].

[27] S.S. Gubser, A. Nellore, S.S. Pufu and F.D. Rocha, Thermodynamics and bulk viscosity of approximate black hole duals to finite temperature quantum chromodynamics, Phys. Rev. Lett. 101 (2008) 131601 [arXiv:0804.1950] [SPIRES]. 